\title{
Stochastic Uncertainty Quantification of Eddy Currents in the Human Body by Polynomial Chaos Decomposition
}

\author{
Roman Gaignaire $^{1}$, Riccardo Scorretti ${ }^{2}$, Ruth V. Sabariego ${ }^{1}$, and Christophe Geuzaine ${ }^{1}$ \\ ${ }^{1}$ Department of Electrical Engineering and Computer Science (ACE), University of Liège, Liège 4000, Belgium \\ ${ }^{2}$ Laboratoire Ampère, Villeurbanne 69622, France
}

\begin{abstract}
The finite element method can be used to compute the electromagnetic fields induced in the human body by environmental extremely low frequency (ELF) fields. However, the electric properties of tissues are not precisely known and may vary depending on the individual, his/her age and other physiological parameters. In this paper, we account for the uncertainties on the conductivities of the brain tissues and spread them out to the induced fields by means of a nonintrusive approach based on Hermite polynomial chaos, with the finite element method as a black box. After showing the convergence of the method, we compute the probability to be over the thresholds defined by the international guidelines for limiting exposure to electromagnetic fields published by ICNIRP.
\end{abstract}

Index Terms-Nonintrusive methods, polynomial chaos decomposition, stochastic methods.

\section{INTRODUCTION}

C URRENT recommendations for avoiding health issues due to over-exposure to ELF radiations may require to evaluate the induced fields in the human body by numerical dosimetry. Unfortunately, these computations are largely affected by the uncertainty on the electric characteristics of the human body, as well as their variability with respect to age and other physiological parameters. Indeed, an arbitrary security factor 3 is considered in [1] in order to account for "dosimetric uncertainties."

Determining the conductivities of the human tissues has been an active research subject for many years [2]. Tissues are highly heterogeneous and possibly anisotropic "materials," [3] the properties of which change rather quickly after death [4] so that measurements performed in vitro on excised tissues may not be representative. Due to ethical reasons, in vivo measurements have been mostly performed on animals. The age [5] and the physiological condition [6] may also significantly alter these properties. Obtaining data for characterising a foetus is even harder [7]. Consequently, at ELF frequencies, the measurements are performed either by identifying an equivalent RC circuit, or by a four-point measurement. At those frequencies, electrode polarization is (another) major source of errors. Some tentatives have been performed [6], [8] for numerically estimating the conductivity and permittivity by modeling tissues as porous media, with limited success. Gabriel et al. [9]-[11] have collected most of the existing data and built a tissue database, which is currently the reference for dosimetric computations [12].

However, the validity of this reference data is still hugely debated, as new measurements by the same authors [13], as well as results obtained using a variety of novel measurement

Manuscript received July 06, 2011; revised October 05, 2011; accepted October 07,2011 . Date of current version January 25, 2012. Corresponding author: C. Geuzaine (e-mail: cgeuzaine@ulg.ac.be) techniques based on magnetic resonance (which can be applied in vivo) [14], [15], show large discrepancies: the new values of conductivities are often higher and muscle-type tissues are found to be much less anisotropic. For example, at $50 \mathrm{~Hz}$, the conductivities of the white and grey matter in [10] and [16] span, respectively, within $[0.0753 ; 0.5155]$ and $[0.0533 ; 0.302] \mathrm{S} / \mathrm{m}$, i.e., differ by more than one order of magnitude.

It is thus, crucial to quantify the effect of this uncertainty on the electromagnetic fields computed in the human body. The classical approach would be to use a time-consuming Monte-Carlo (MC) simulation to statistically characterise the induced fields - which would take several weeks of computational time for our models. In this paper, we use a polynomial chaos approach, the so-called nonintrusive probabilistic algorithm, which assumes that the variances of conductivities are finite [17], [18] and allows to completely characterize the induced field in the probabilistic dimension with a much lower computational cost (a few hours). We then use this characterization to compute the probability to be over the thresholds defined by the international guidelines for limiting exposure to electromagnetic fields published by ICNIRP [1], [19].

\section{INCORPORATION OF STOCHASTIC UNCERTAINTY}

\section{A. Deterministic Framework}

The fields induced in the human body are computed by the finite element method using a $\phi-\mathbf{a}$ formulation. Details of this formulation and the phantom used can be found in [20]: see Fig. 1. We simulate the exposure to the field generated by an infinite cable (current $I=1000 \mathrm{~A}$ at $50 \mathrm{~Hz}$ ) placed at a few centimeters from the left side of the head. In the literature on the protection against ELF fields, three global quantities are generally computed for each organ from the induced current density: the (spatial) average $j_{\mathrm{Avg}}$, the maximum value $j_{\max }$, and the $99 \%$ percentile $j_{99-\text { perc }}$ [21]. Similar definitions exist for the electric field. The basic restriction of the 1998 edition of the ICNIRP recommendations [19] is based on $j_{\max }$, while the 2010 edition [1] focuses on $e_{99-p e r c}$. In particular, for a $50 \mathrm{~Hz}$ occupational exposure the threshold values in the central 

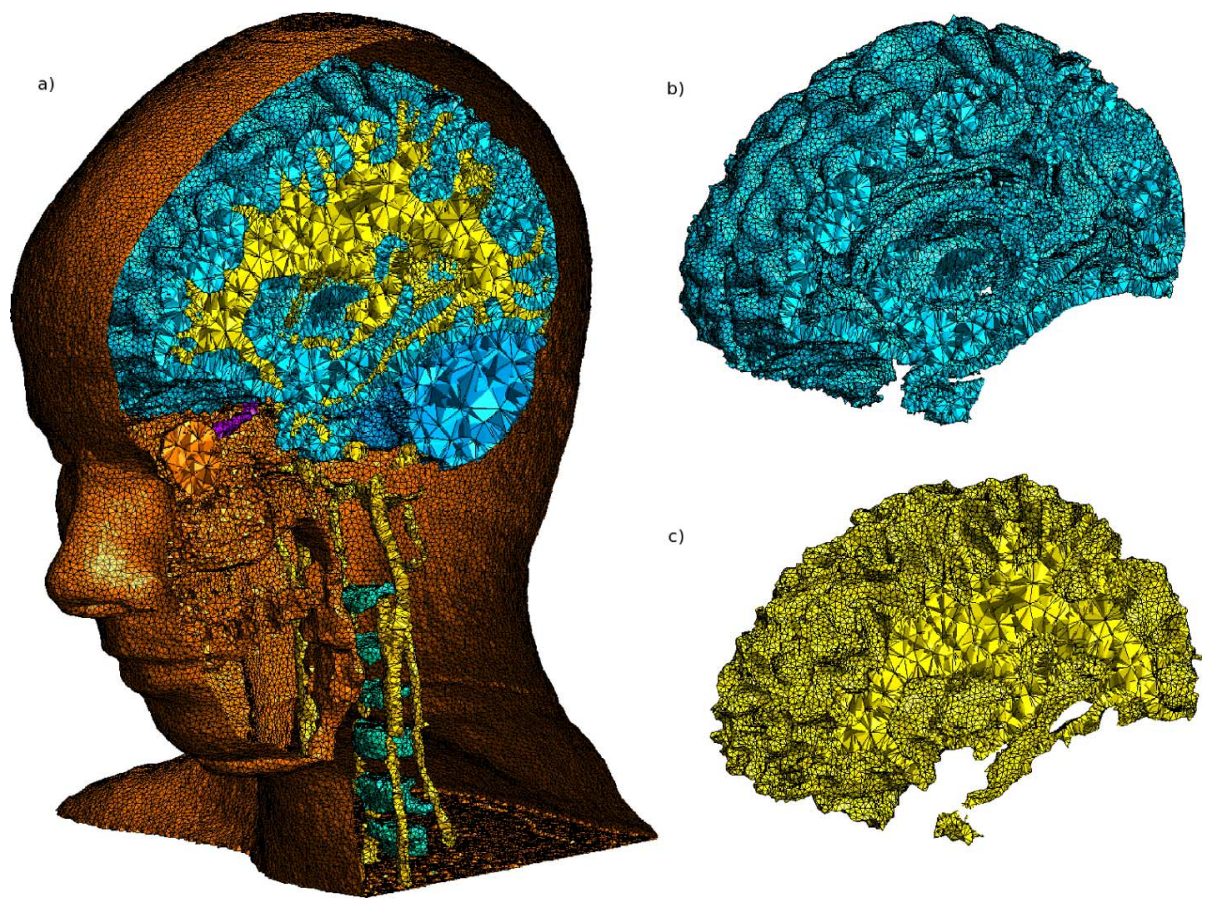

Fig. 1. Mesh used for the numerical experiments [20]: (a) full head (300 000 nodes, 27 tissues); (b) grey matter; (c) white matter.

nervous system (CNS) recommended by ICNIRP are $j_{\max }<10$ $\mathrm{mA} / \mathrm{m}^{2}$ and $e_{99-\text { perc }}<100 \mathrm{mV} / \mathrm{m}$.

\section{B. Uncertainties}

Let us focus on the fields induced in the brain. The conductivities of the white matter $\sigma_{W}(\omega)$ and of the grey matter $\sigma_{G}(\omega)$ are modeled within a probabilistic framework, as functions of the random variable $\omega$. Therefore $j_{\mathrm{Avg}}, j_{\max }, j_{99-\mathrm{perc}}, e_{\mathrm{Avg}}, e_{\max }$, and $e_{99-\text { perc }}$ are random as well. In particular, by using the maximum entropy principle [22] we model (arbitrarily) $\sigma_{G}(\omega)$ and $\sigma_{W}(\omega)$ as independent random variables, uniformly distributed

$$
\begin{aligned}
\sigma_{G}(\omega) & \sim \mathrm{U}([0.0753 ; 0.5155])(\mathrm{S} / \mathrm{m}) \\
\sigma_{W}(\omega) & \sim \mathrm{U}([0.0533 ; 0.3020])(\mathrm{S} / \mathrm{m}) .
\end{aligned}
$$

\section{The Nonintrusive Approach}

As the conductivities of the brain and the cerebellum are two independent random variables of finite variance, we can expand them as a truncated series of order $p_{\text {in }}$ in the bidimensional Hermite polynomials of a random gaussian vector $\xi(\omega)=$ $\left(\xi_{1}(\omega), \xi_{2}(\omega)\right)$, known as Hermite chaos polynomials [18]

$$
\begin{aligned}
\sigma_{G}(\omega) & \approx \sum_{i=0}^{P_{\text {in }}} \sigma_{G i} \psi_{i}(\xi(\omega)) \\
\sigma_{W}(\omega) & \approx \sum_{i=0}^{P_{\text {in }}} \sigma_{W i} \psi_{i}(\xi(\omega))
\end{aligned}
$$

where $\sigma_{G i}$ and $\sigma_{W i}$ are scalar values that depend on the probabilistic law of the conductivities, $P_{\text {in }}=C_{2+p_{\text {in }}}^{p_{\text {in }}}$ is the number of bidimensional polynomials of order less than $p_{\text {in }}$, and $\psi_{i}$ is the $i$ th bidimensional Hermite polynomial. To solve the stochastic problem, we use an approach based on a polynomial chaos decomposition of both the conductivity and the induced fields [18]. We assume the conductivities to be of finite variance, with no assumption on the shape of the probabilistic distribution.

The values of the induced fields-the average current density in the brain $j_{\mathrm{Avg}}(\omega)=j_{\mathrm{Avg}}(\xi(\omega))$-are computed by the finite element method from any couple of values $\left(\sigma_{G}(\xi(\omega)), \sigma_{W}(\xi(\omega))\right)$. The average density belongs to a space that can be spanned by the polynomials $\psi(\xi(\omega))$ and thus written as a truncated series to an order $p_{\text {out }}$

$$
j_{\mathrm{Avg}}(\omega)=\sum_{m=0}^{P_{\text {out }}} j_{\operatorname{Avg}_{m}} \psi_{m}(\xi(\omega)) \text {. }
$$

To compute the value of the unknown real coefficients $j_{\mathrm{Avg}}$, we use the orthogonality properties of the Hermite polynomials

$$
j_{\mathrm{Avg} m}=\frac{\mathrm{E}\left[j_{\mathrm{Avg}}(\omega) \psi_{m}(\xi(\omega))\right]}{\mathrm{E}\left[\psi_{m}(\xi(\omega))^{2}\right]}
$$

where $\mathrm{E}[\cdot]$ is the mathematical expectation. The denominator can be computed analytically. The integral in the numerator is computed by means of a Hermite Gauss integration scheme with $d$ integration points [18]

$$
\begin{aligned}
& \mathrm{E}\left[j_{\operatorname{Avg}}(\omega) \psi_{m}(\xi(\omega))\right] \\
& \quad \approx \sum_{i=1}^{d} \cdots \sum_{j=1}^{d} w_{i, j}\left(j_{\operatorname{Avg}}\left(\left(t_{1}, t_{2}\right)_{i, j}\right)\right) \psi_{m}\left(\left(t_{1}, t_{2}\right)_{i, j}\right)
\end{aligned}
$$

with $\left(t_{1}, t_{2}\right)_{i, j}$ the $i, j$-th Gauss point and $w_{i, j}$ the associated weight in the bidimensional Cartesian rule. The deterministic problem must thus be computed $d^{2}$ times, with the conductivity evaluated through (3) and $\left(\xi_{1}(\omega), \xi_{2}(\omega)\right)=\left(t_{1},=t_{2}\right)_{i, j}, i, j=$ $1 \ldots d$. 


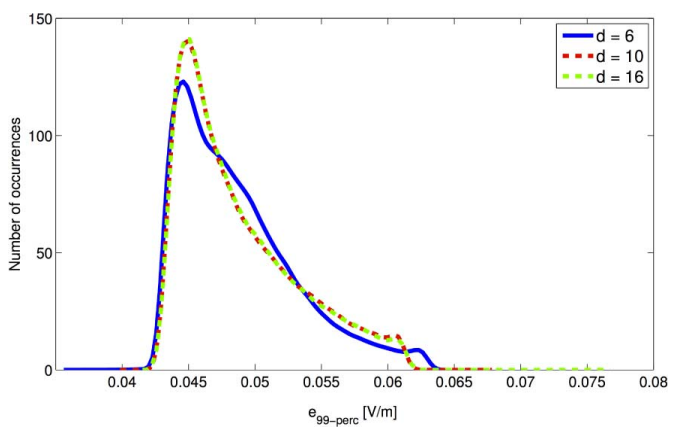

Fig. 2. Probability density of $e_{99-\text { perc }}$ in the grey matter for $p_{\text {out }}=8, p_{\text {in }}=$ 16 and $d=6$ (blue), $d=10$ (red), $d=16$ (green).

\section{RESUlTS AND DisCUSSION}

The non intrusive method is governed by three parameters: $p_{\text {in }}, p_{\text {out }}$ and $d ; p_{\text {in }}$ is linked to the precision on the approximation made on the input random variables $\sigma_{G}(\omega)$ and $\sigma_{W}(\omega)$, $p_{\text {out }}$ is the order of truncation of the studied global quantities $\left(j_{\mathrm{Avg}}, j_{\max }, j_{99-\text { perc }}\right.$ and the corresponding quantities for the electric field) and $d$ is the number of quadrature points. Herein, we have chosen $p_{\text {in }}=16$, while $p_{\text {out }}$ and $d$ vary. For the sake of conciseness, we deal with the white and grey matter (though the method could handle other tissues during the same computation as well).

\section{A. Influence of the Input Parameters}

The probability density (PD) of $e_{99-\text { perc }}$ in the grey matter obtained with $p_{\text {out }}=8, p_{\text {in }}=16$ and different values of $d$ is shown in Fig. 2. The curves of the PD obtained with $d=10$ and $d=16$ are nearly superposed, which proves the convergence of the method with increasing values of $d$.

Concerning dispersion parameters as the mean and the standard deviation, the convergence is reached as soon as $d \geq 4$ (mean: $0.0487 \mathrm{~V} / \mathrm{m}$, standard deviation: 0.07 when $d=2$ converges to 0.0044 for $d=6$ ).

The PD of $j_{\max }$ in the white matter obtained with $d=16$, $p_{\text {in }}=16$ and different values of $p_{\text {out }}$ are plotted in Fig. 3 . Again, one observes that convergence is achieved as $p_{\text {out }}$ increases. The value of $p_{\text {out }}$ has a minor influence on the central dispersion parameters (mean and variance): the mean is constant and equal to $13.1 \mathrm{~mA} / \mathrm{m}^{2}$, and the standard deviation converges with $p_{\text {out }} \geq 3$ to $3.4 \mathrm{~mA} / \mathrm{m}^{2}$. It can be observed that the support of the PD is bounded by $21 \mathrm{~mA} / \mathrm{m}^{2}$ for $p_{\text {out }} \geq 4$ (i.e., most likely $j_{\max }<21 \mathrm{~mA} / \mathrm{m}^{2}$ ): conversely, $p_{\text {out }}=2$ would lead to the wrong conclusion that $j_{\max }$ may exceed $21 \mathrm{~mA} / \mathrm{m}^{2}$ with a nonnegligible probability.

\section{B. Analysis of the Results}

The PD of $j_{\mathrm{Avg}}, j_{99-\text { perc }}$ and $j_{\max }$ linked to the induced current density in the grey matter are represented in Fig. 4 for $p_{\text {out }}=4$ and $p_{\text {out }}=8$. (For $p_{\text {out }}=6$, the curves are very close to those for $p_{\text {out }}=8$, which proves that the method has converged.) The PD of $e_{\mathrm{Avg}}, e_{99-\text { perc }}$ and $e_{\max }$ related to the electric field in the white matter are depicted in Fig. 5 for $p_{\text {out }}=4$ and $p_{\text {out }}=8$. These PDs are more "peaked" than those corresponding to the current density, i.e., they are less dispersed around their means. Moreover, the area under these curves for

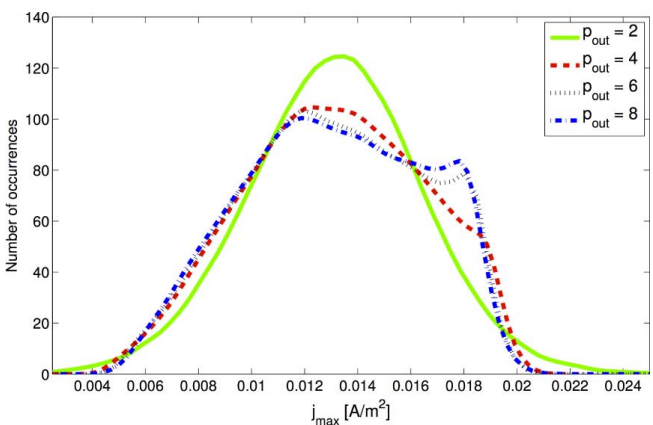

Fig. 3. Probability density of $j_{\max }$ in the white matter for $d=16, p_{\text {in }}=16$ and $p_{\text {out }}=2$ (black), $p_{\text {out }}=4$ (red), $p_{\text {out }}=6$ (green), $p_{\text {out }}=8$ (blue).

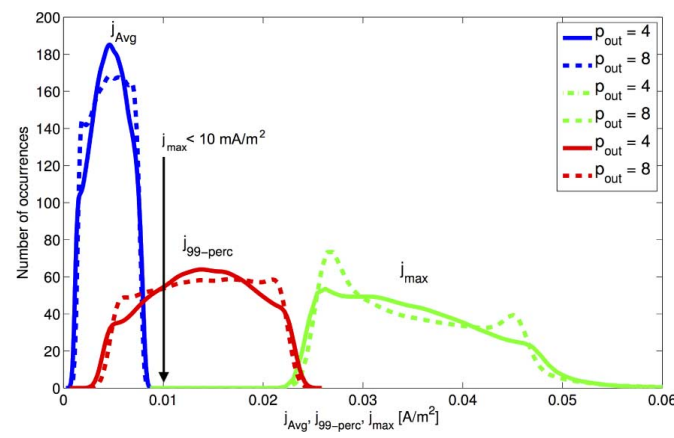

Fig. 4. Probability density of $j_{\mathrm{Avg}}$ (blue), $j_{99-\operatorname{perc}}$ (red) and $j_{\max }$ (green) in the grey matter $\left(p_{\text {in }}=16, d=16\right)$.

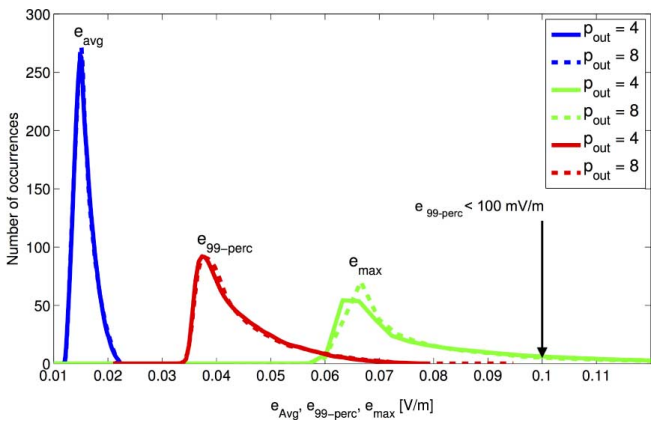

Fig. 5. Probability density of $e_{\mathrm{Avg}}$ (blue), $e_{99-\text { perc }}$ (red) and $e_{\max }$ (green) in the white matter $\left(p_{\text {in }}=16,=16\right)$.

$e>100 \mathrm{mV} / \mathrm{m}$ equals 0 for $e_{\mathrm{Avg}}$ and $e_{99-\text { perc }}$ and nearly 0 for $e_{\max }$.

In order to avoid health hazards, ICNIRP recommends that in the central nervous system $j_{\max } \leq 10 \mathrm{~mA} / \mathrm{m}^{2}$ [19] or $e_{99-\text { perc }} \leq 100 \mathrm{mV} / \mathrm{m}$ [1]. As these global quantities are available as a polynomial expansion like (5), we can estimate the probability $p$ that these recommendations are not fulfilled-e.g., in the case of [19], $p$ is: $\mathcal{P}\left\{j_{\max } \leq 10 \mathrm{~mA} / \mathrm{m}^{2}\right\}$. To this aim, a large number $n$ of couples of independent values following a normal variable $\left(\xi_{1 i}, \xi_{2 i}\right)_{1 \leq i \leq 10^{4}}$ are sampled. The polynomial expansion (5) is evaluated for each pair of values and the number of occurrences (i.e., the number of values of $j_{\max }>10 \mathrm{~A} / \mathrm{m}^{2}$ ) for which the basic restriction is exceeded are counted. The probability $p$ and the confidence interval CI are estimated by means of the central limit theorem as

$$
p=\frac{\text { Number of occurrences }}{n}
$$


TABLE I

PROBABILITY TO EXCEED THE ICNIRP THRESHOLDS

\begin{tabular}{ccc}
\hline \hline Field & Grey matter & White matter \\
\hline$e_{\text {avg }}$ & $0 \pm 0$ & $0 \pm 0$ \\
$e_{99-\text { perc }}$ & $0 \pm 0$ & $0 \pm 0$ \\
$e_{\text {max }}$ & $0.8 \pm 2.7 \times 10^{-3}$ & $0.14 \pm 2.2 \times 10^{-3}$ \\
\hline$j_{\text {avg }}$ & $0 \pm 0$ & $0 \pm 0$ \\
$j_{99-\text { perc }}$ & $0.73 \pm 2.8 \times 10^{-3}$ & $0.16 \pm 2.3 \times 10^{-3}$ \\
$j_{\text {max }}$ & $1 \pm 0$ & $0.80 \pm 2.5 \times 10^{-3}$ \\
\hline \hline
\end{tabular}

$$
\mathrm{CI}=z_{.05 / 2} \sqrt{\frac{p(1-p)}{n}}
$$

where $z_{.05 / 2}$ is the confidence interval of $97.5 \%$ of a normal random variable (i.e., there is a risk of $5 \%$ that the true probability is outside the confidence interval). The cost of this computation is negligible with regard to the coefficient evaluation via (6).

The probability that each of the global quantities exceeds the ICNIRP thresholds for the simulated exposure has been computed with $n=10^{5}$ and the obtained values are reported in Table I. (For instance, the third line in Table I reads: the probability for $e_{\max }>100 \mathrm{mV} / \mathrm{m}$ is of $80 \% \pm 0.27 \%$ with a risk of $5 \%$.)

According to the most recent ICNIRP recommendations [1], the threshold $e_{99-\text { perc }} \leq 100 \mathrm{mV} / \mathrm{m}$ is most likely fulfilled-it would not have been the case if we considered $e_{\max }$ instead of $e_{99-\text { perc }}$. Concerning the 1998 ICNIRP recommendations [19], the threshold $j_{\max } \leq 10 \mathrm{~mA} / \mathrm{m}^{2}$ is clearly not fulfilled for the grey matter (see also Fig. 4), while for the white matter the probability for $j_{\max }$ to exceed $10 \mathrm{~mA} / \mathrm{m}^{2}$ is of $80 \%$. These probabilities decrease respectively to $73 \%$ and $16 \%$ when dealing with $j_{99-\text { perc }}$ instead of $j_{\text {max }}$.

\section{CONCLUSION}

The proposed recommendations for avoiding health issues due to over-exposure to ELF radiations may require to evaluate the induced fields in the human body by dosimetric methods. Unfortunately, these computations are largely affected by the uncertainty on the conductivities of human tissues. In this paper we proposed an effective method for quantifying the uncertainty on some relevant quantities (notably $j_{\max }$ and $e_{99-\text { perc }}$ ), provided that a characterisation of the statistical distribution of the conductivities is available. Our simulations suggest that the latest 2010 ICNIRP recommendations are more permissive than those in the former edition. However, it has to be pointed out that in our computations we use a quite pessimistic statistical law for the conductivities, and also that other sources of uncertainty (posture, physiognomy, etc.) are disregarded.

Finally, note that our computational scheme is only effective for a small number of uncertain parameters. With many uncertain parameters (up to 30 in modern dosimetric computations), the proposed approach could be accelerated by reducing the number of stochastic dimensions by means of the Principal Component Analysis and a sparse (Smolyak) quadrature.

\section{ACKNOWLEDGMENT}

This work was been supported in part by the Belgian French Community (ARC 09/14-02), the Belgian Science Policy (IAP P6/21), and the Belgian BioElectroMagnetic Group (BBEMG) EMF program.

\section{REFERENCES}

[1] ICNIRP, "Guidelines for limiting exposure to time-varying electric and magnetic fields (1 Hz to $100 \mathrm{kHz}$ )," Health Phys., vol. 99, no. 6, pp. 818-836, 2010.

[2] L. Geddes and L. Baker, "The specific resistance of biological material-A compendium of data for the biomedical engineer and physiologist," Med. Biol. Eng. Comput., vol. 5, no. 3, pp. 271-293, 1967.

[3] F. Sachse, M. Wolf, C. Werner, and K. Meyer-Waarden, "Extension of anatomical models of the human body: Three-dimensional interpolation of muscle fiber orientation based on restrictions," J. Comp. Inf. Tech., vol. 6, no. 1, pp. 95-101, 1998.

[4] D. Haemmerich, O. Ozkan, J. Tsai, S. Staelin, S. Tungjitkusolmun, D. Mahvi, and J. Webster, "Changes in electrical resistivity of swine liver after occlusion and postmortem," Med. Biol. Eng. Comput., vol. 40, no. 1, pp. 29-33, 2002.

[5] C. Gabriel, "Dielectric properties of biological tissue: Variation with age," Bioelectromagn., vol. 26, no. S7, pp. S12-S18, 2005.

[6] C. Peratta et al., "Modelling the human body exposure to elf electric fields," Medical Phys., vol. 37, p. 5561, 2010.

[7] R. Cech, N. Leitgeb, and M. Pediaditis, "Fetal exposure to low frequency electric and magnetic fields," Phys. Med. Biol., vol. 52, p. 879, 2007.

[8] S. W. Smye, C. J. Evans, M. P. Robinson, and B. D. Sleeman, "Modelling the electrical properties of tissue as a porous medium," Phys. Med. Biol., vol. 52, no. 23, pp. 7007-7022, Dec. 2007.

[9] C. Gabriel, S. Gabriel, and E. Corthout, "The dielectric properties of biological tissues: I. Literature survey.," Phys. Med. Biol., vol. 41, no. 11, pp. 2231-2249, Nov. 1996.

[10] S. Gabriel, R. W. Lau, and C. Gabriel, "The dielectric properties of biological tissues: II. Measurements in the frequency range $10 \mathrm{~Hz}$ to 20 GHz.," Phys. Med. Biol., vol. 41, no. 11, pp. 2251-2269, Nov. 1996.

[11] S. Gabriel, R. W. Lau, and C. Gabriel, "The dielectric properties of biological tissues: III. Parametric models for the dielectric spectrum of tissues.," Phys. Med. Biol., vol. 41, no. 11, pp. 2271-2293, Nov. 1996.

[12] R. F. D. Andreuccetti, "An internet resource for the calculation of the dielectric properties of body tissues," [Online]. Available: http://niremf.ifac.cnr.it/tissprop

[13] C. Gabriel, A. Peyman, and E. H. Grant, "Electrical conductivity of tissue at frequencies below $1 \mathrm{MHz}$," Phys. Med. Biol., vol. 54, no. 16, pp. 4863-4878, 2009.

[14] M. Sekino, Y. Inoue, and S. Ueno, "Magnetic resonance imaging of electrical conductivity in the human brain," IEEE Trans. Magn., vol. 41, no. 10, pp. 4203-4205, Oct. 2005.

[15] M. Sekino, H. Ohsaki, S. Yamaguchi-Sekino, N. Iriguchi, and S. Ueno, "Low-frequency conductivity tensor of rat brain tissues inferred from diffusion MRI," Bioelectromagnetics, vol. 30, no. 6, pp. 489-499, 2009.

[16] J. Latikka, T. Kuurne, and H. Eskola, "Conductivity of living intracranial tissues," Phys. Med. Biol., vol. 46, p. 1611, 2001

[17] R. Gaignaire, G. Crevecoeur, L. Dupré, R. Sabariego, P. Dular, and C. Geuzaine, "Stochastic uncertainty quantification of the conductivity in eeg source analysis by using polynomial chaos decomposition," IEEE Trans. Magn., vol. 46, no. 8, pp. 3457-3460, Aug. 2010.

[18] R. Gaignaire, O. Moreau, B. Sudret, and S. Clénet, "Propagation d'incertitudes en électromagnétisme statique par chaos polynomial et résolution non intrusive," in Proc. NUMELEC 2006, Lille, 2006.

[19] ICNIRP, "Guidelines for limiting exposure to time-varying electric, magnetic, and electromagnetic fields (up to 300 ghz)," Health Phys., vol. 74, no. 4, pp. 494-522, 1998.

[20] R. Scorretti, R. Sabariego, L. Morel, C. Geuzaine, N. Burais, and L. Nicolas, "Computation of induced fields into the human body by dual finite element formulations," IEEE Trans. Magn., 2011, to be published.

[21] P. J. Dimbylow, "Induced current densities from low-frequency magnetic fields in a $2 \mathrm{~mm}$ resolution, anatomically realistic model of the body," Phys. Med. Biol., vol. 43, no. 2, pp. 221-230, Feb. 1998.

[22] E. Jaynes, "Information theory and statistical mechanics," Phys. Rev., vol. 108, no. 2, p. 171, 1957. 